\title{
EMPIRICAL VERSUS SUSCEPTIBILITY-BASED ERADICATION THERAPY FOR HELICOBACTER PYLORI INFECTION IN EGYPT
}

\author{
By
Amr El-Gazzar, Mohamed Al-Boraie, Sawsan Hanem El-Tayyeb* and Adel El-Rakeeb

Departments of Internal Medicine and Clinical Pathology*, Al-Azhar Faculty of Medicine

E-mail: amr.elgazzar01@gmail.com

\begin{abstract}
Background: The eradication rate of Helicobacter pylori has declined, mainly due to antimicrobial resistance.

Objectives: To test the effectiveness of susceptibility guided therapy vs. the empirical triple therapy for first: line $\mathbf{H}$ pylori treatment in a region with high antimicrobial resistance.

Patients and methods: The study was performed on 82 dyspeptic H. pylori patients arranged for esophagogastroduodenoscopy at Al-Hussein University Hospital between March 2019 to march 2020. Patients were randomly divided into two groups: group A consisted of 52 patients underwent endoscopy and gastric biopsies for culture and sensitivity and treated according to sensitivity results and group B consisted of 30 patients treated by empiric triple therapy. Eradication rates and drug compliance owing to adverse effects were compared between the two groups.

Results: In total, 82 patients were enrolled (52 in culture and sensitivity-based therapy group and 30 in empirical therapy group) and 50 patients $(60.1 \%)$ completed the protocols. The overall resistance rates to clarithromycin, amoxicillin, metronidazole, levofloxacin, tetracycline, nitrofurantoin and rifampicin were $32.1 \%, 32.1 \%, 78.6 \%, 7.1 \%, 17.9 \%, 17.9 \%$ and $21.4 \%$ respectively. Empirical triple and antimicrobial susceptibility-guided eradication rates were, respectively, $53.3 \%$ and $85.7 \%$ by intention-to-treat and $64 \%$ and $96 \%$ by per-protocol analysis. Adverse events were reported in $16.6 \%$ of patients on empirical triple therapy and $21.4 \%$ of those on susceptibility-guided therapy.
\end{abstract}

Conclusions: Culture-based eradication strategy demonstrated superior eradication efficacy than empirical therapy as a first-line therapy in a region with high levels of antimicrobial resistance.

Keywords: Antibiotics, eradication, Helicobacter pylori, resistance, clarithromycin.

\section{INTRODUCTION}

Helicobacter pylori (H. pylori) is one of the most common human pathogens affecting more than half of the world's population (Chey et al., 2017 and Malfertheiner et al., 2017).

H. pylori cause gastritis, gastric ulcers, and neoplastic diseases that include mucosa- associated lymphoid tissue lymphoma (MALT lymphoma) and gastric cancer. $\mathrm{H}$ pylori eradication might improve the outcomes of the related diseases, complications, and relapses (Fallone et al., 2016).

Unfortunately, the $\mathrm{H}$ pylori eradication rates have decreased to unacceptable low levels in many regions because of increased antibiotic resistance especially to clarithromycin (Camargo et al., 2014 
and Thung et al., 2016). Thus the conventional clarithromycin triple therapy should not be used in areas of high clarithromycin resistance as adopted by the majority of guidelines, i.e. The Maastricht V/Florence Consensus, Kyoto global consensus and American college of gastroenterology guidelines (Chey et al., 2017 and Malfertheiner et al., 2017).

Consensus statements recommend alternative treatment regimens to the standard triple therapy as first- line therapies; these include sequential, concomitant, or bismuth containing quadruple therapy in regions with high levels of antimicrobial resistance (Fallone et al., 2016 and Malfertheiner et al., 2017). These alternative treatments usually consist of three (partly unnecessary) antibiotics, thus promoting the misuse of antibiotics which fuels antimicrobial resistance (Dang et al., 2017). However, antimicrobial susceptibility- guided therapy could avoid such problems if applied as a first-line treatment.

Culture-based, susceptibility- guided therapy is a well-established strategy. It can prevent resistance-associated treatment failure and the emergence of antibiotic resistance, including multidrug resistance (Lee et al., 2019).

In this study, we aimed to compare the efficacy of pretreatment antimicrobial susceptibility-guided vs. the empiric triple therapy for $\mathrm{H}$. pylori eradication.

\section{PATIENTS AND METHODS}

This study was designed as a prospective and observational study. A total of 82 consecutive $H$. pylori positive patients (out of 155 screened patients) with dyspeptic symptoms and without previous $H$. pylori treatment were included in this clinical study from March 2019 to March 2020. All patients underwent esophagogastroduodenoscopy at Al-Hussein University Hospital, Department of Internal Medicine, Gastroenterology and Hepatology Unit. H. pylori infection was defined by a positive rapid urease test.

Exclusion criteria included history of previous H. pylori eradication therapy, under 18 years old, pregnant or lactating women, previous gastric surgery, gastrointestinal bleeding, severe concomitant cardiovascular, respiratory, renal, hepatic and other diseases precluding study therapy, treatment with proton pump inhibitors, histamine2blockers and/or antibiotics during the 4 weeks before the study or allergy to any of the drugs used in the study.

Patients enrolled in this study were divided into two groups:

Group A composed of 28 patients with positive cultures (of 52 patients allocated randomly for culture guided therapy). They were treated after successful culture according to antibiotic sensitivity results.

Group B composed of 30 patients. They were selected randomly and treated empirically according to the current international recommendations .

One gastric biopsy was taken at endoscopy and tested for the presence of H. pylori by rapid urease test (CLO test). Four biopsy specimens (two antral and two corpuses) were taken, stored in $0.3 \mathrm{ml}$ normal saline, and immediately sent to the microbiology laboratory for $H$. pylori culture. Biopsy specimens were cultured and maintained on brain heart infusion 
agar medium containing 5\% defibrinated sheep blood under microaerophilic conditions $(85 \%$ nitrogen, $10 \%$ carbon dioxide and $5 \%$ oxygen) at $37^{\circ} \mathrm{C}$. If $\mathrm{H}$. pylori were not isolated after 7 days of incubation, the plates were incubated for 14 days. Culture was considered positive if one or more colonies were gramnegative with spiral or curved rods and positive for urease, oxidase and catalase.

The disk diffusion method was used to detect the sensitivity to 7 antibiotics; clarithromycin, amoxicillin, metronidazole, levofloxacin, tetracycline, nitrofurantoin and rifampicin. All inhibitory zones were interpreted using CLSI (Clinical and Laboratory Standards Institute) clinical breakpoints.

As regards to the empirical therapy group, patients were given $20 \mathrm{mg}$ esomeprazole, amoxicillin 1gram and clarithromycin $500 \mathrm{mg}$ twice daily for 14 days. Patients who were previously exposed to clarithromycin for any cause were given levofloxacin based triple therapy (20 mg esomeprazole, amoxicillin 1 gram twice daily and levofloxacin 500 $\mathrm{mg}$ once daily for 14 days).

At least 4 weeks after completion of therapy, $\mathrm{H}$ pylori eradication was confirmed by negative $\mathrm{H}$ pylori stool antigen test. All patients were asked to stop antibiotics, bismuth, PPIs, histamine2 blockers for at least 4 weeks before the H. pylori stool antigen test. Additional outcomes included the frequency of adverse events, and adherence rates. The study design was approved by the ethics and scientific research committee of Faculty of Medicine, Al-Azhar University. Informed consents were obtained from all patients. The study protocol confers with the principals of the declaration of Helsinki.

\section{Statistical Analysis:}

We estimated the sample size needed to detect a difference of $31 \%$ in the eradication rate between the susceptibility-guided therapy (assumed to have an eradication rate of $96 \%$ ) and the standard triple therapy groups (assumed to have an eradication rate of $65 \%$ ), with a power of $80 \%$ and a confidence level of $95 \%$. The required sample size was at least 25 patients per group. Taking into consideration of $10 \%$ lost to follow- up, at least 56 subjects (28 for susceptibility- guided therapy and 28 for empiric therapy) were expected to be recruited for the study.

Intention-to-treat and per-protocol analyses were performed to compare $\mathrm{H}$ pylori eradication rates in the two groups. Categorical variables are reported as numbers and percentages and compared using the chi-square test. Continuous variables were presented as means \pm SD (standard deviation) and compared by (Mann-whiney U test). All statistical analyses were performed using SPSS (version 21.0). P-value $\leq 0.05$ was considered significant. 


\section{RESULTS}

In total, 82 patients met the inclusion criteria and agreed to participate in our study. 52 patients were allocated randomly to susceptibility-based therapy group and 30 patients were allocated to the empirical therapy group. Twenty four withdrawals $(46.2 \%)$ subsequently occurred in the culture-based tailored therapy owing to culture failure. The remaining $28(53.8 \%)$ patients were assigned to the treatment group according to the susceptibility results. One patient stopped treatment due to medication side effects, and two patients were lost to follow- up. Finally, 25 patients completed the culture based therapy and 24 patients were successfully eradicated.

In the empirical therapy group, one patient stopped treatment due to medication side effects, and four patients were lost to follow up. Finally, 25 patients completed the empirical therapy and 16 patients were successfully eradicated. There were no significant differences between the groups in terms of age, gender, smoking, comorbidities and clinical manifestations (Table 1).

Antimicrobial susceptibility testing using the disk diffusion methods demonstrated high rates of resistance to metronidazole, clarithromycin, amoxicillin $(78.6 \%, 32.1 \%$, and $32.1 \%$ respectively), while levofloxacin resistance was as low as $7.1 \%$. Resistance rates to tetracycline, nitrofurantoin and rifampicin were $17.9 \%, 17.9 \%$, and $21.4 \%$ respectively (Table 2). 
EMPIRICAL VERSUS SUSCEPTIBILITY-BASED ERADICATION...

Table (1): Baseline characteristics

\begin{tabular}{|c|c|c|c|c|c|c|}
\hline \multicolumn{2}{|c|}{$\begin{array}{ll}\text { Parameters } & \text { Groups }\end{array}$} & \multicolumn{2}{|c|}{$\begin{array}{c}\text { Group A } \\
(\mathbf{N}=\mathbf{2 8})\end{array}$} & \multicolumn{2}{|c|}{$\begin{array}{c}\text { Group B } \\
(\mathbf{N}=\mathbf{3 0}) \\
\end{array}$} & \multirow{2}{*}{$\begin{array}{c}\text { P-value } \\
\mathrm{MW}=337 \\
\mathrm{P}=0.367\end{array}$} \\
\hline Age (years) & Mean \pm SD & \multicolumn{2}{|c|}{$37.4 \pm 14.5$} & \multicolumn{2}{|c|}{$40.7 \pm 12.9$} & \\
\hline \multirow{2}{*}{ Sex } & Males & $(9 / 28)$ & $32.1 \%$ & $(12 / 30)$ & $40 \%$ & \multirow{2}{*}{0.534} \\
\hline & Females & $(19 / 28)$ & $67.9 \%$ & $(18 / 30)$ & $60 \%$ & \\
\hline \multirow{2}{*}{ Smoking } & Non-smokers & $(22 / 28)$ & $78.6 \%$ & $(22 / 30)$ & $73.3 \%$ & \multirow{2}{*}{0.641} \\
\hline & Smokers & $(6 / 28)$ & $21.4 \%$ & $(8 / 30)$ & $26.7 \%$ & \\
\hline \multirow{5}{*}{$\begin{array}{l}\text { Comorbidi } \\
\text { ties }\end{array}$} & Non & $(21 / 28)$ & $75 \%$ & $(22 / 30)$ & $73.3 \%$ & \multirow{5}{*}{0.839} \\
\hline & Diabetics & $(3 / 28)$ & $10.7 \%$ & $(4 / 30)$ & $13.3 \%$ & \\
\hline & Hypertensive & $(2 / 28)$ & $7.1 \%$ & $(2 / 30)$ & $6.7 \%$ & \\
\hline & $\begin{array}{l}\text { Chronic liver } \\
\text { disease }\end{array}$ & $(1 / 28)$ & $3.6 \%$ & $(2 / 30)$ & $6.7 \%$ & \\
\hline & Crohn's disease & $(1 / 28)$ & $3.6 \%$ & $(0 / 30)$ & $0 \%$ & \\
\hline \multirow{2}{*}{$\begin{array}{c}\text { Epigastric } \\
\text { pain }\end{array}$} & No & $(5 / 28)$ & $17.9 \%$ & $(4 / 30)$ & $13.3 \%$ & \multirow{2}{*}{0.634} \\
\hline & Yes & $(23 / 28)$ & $82.1 \%$ & $(26 / 30)$ & $86.7 \%$ & \\
\hline \multirow{2}{*}{ Vomiting } & No & $(14 / 28)$ & $50 \%$ & $(17 / 30)$ & $56.7 \%$ & \multirow{2}{*}{0.611} \\
\hline & Yes & $(14 / 28)$ & $50 \%$ & $(13 / 30)$ & $43.3 \%$ & \\
\hline \multirow{2}{*}{ Bloating } & No & $(19 / 28)$ & $67.9 \%$ & $(20 / 30)$ & $66.7 \%$ & \multirow{2}{*}{0.923} \\
\hline & Yes & $(9 / 28)$ & $32.1 \%$ & $(10 / 30)$ & $33.3 \%$ & \\
\hline \multirow{2}{*}{ Anorexia } & No & $(21 / 28)$ & $75 \%$ & $(23 / 30)$ & $76.7 \%$ & \multirow{2}{*}{0.882} \\
\hline & Yes & $(7 / 28)$ & $25 \%$ & $(7 / 30)$ & $23.3 \%$ & \\
\hline
\end{tabular}

Table (2): H. pylori antimicrobial resistance rates

\begin{tabular}{|c|c|c|}
\hline \multirow{2}{*}{ Antibiotics } & \multicolumn{2}{|c|}{ Resistance rate (n/N) \% } \\
\hline Clarithromycin & $(9 / 28)$ & $32.1 \%$ \\
\hline Amoxicillin & $(9 / 28)$ & $32.1 \%$ \\
\hline Metronidazole & $(22 / 28)$ & $78.6 \%$ \\
\hline Levofloxacin & $(2 / 28)$ & $7.1 \%$ \\
\hline Tetracycline & $(5 / 28)$ & $17.9 \%$ \\
\hline Nitrofurantoin & $(5 / 28)$ & $17.9 \%$ \\
\hline Rifampicin & $(6 / 28)$ & $21.4 \%$ \\
\hline
\end{tabular}

In the empiric therapy group; clarithromycin-based triple therapy was given to 19 patients, while levofloxacinbased triple therapy was given to 11 patients who had a history of previous exposure to clarithromycin. One patient stopped treatment due to medication side effects, and four patients were lost to follow- up. Finally, 25 patients completed the empirical therapy and 16 patients were successfully eradicated. Levofloxacinbased triple therapy demonstrated better eradication rates than clarithromycinbased triple therapy (Table 3). 
Table (3): Outcomes in empiric therapy group

\begin{tabular}{|c|c|c|c|}
\hline $\begin{array}{r}\text { Eradication } \\
\text { rates }\end{array}$ & $\begin{array}{c}\text { Eradication rate \% } \\
\text { ITT analysis (N=30) }\end{array}$ & $\begin{array}{c}\text { Eradication rate \% } \\
\text { PP analysis (N=25) }\end{array}$ & $\begin{array}{c}\text { Overall } \\
\text { eradication rate } \\
\text { PP analysis }\end{array}$ \\
\cline { 1 - 3 } $\begin{array}{c}\text { Clarithromycin } \\
\text { triple therapy }\end{array}$ & $(9 / 19)$ & $(9 / 16)$ & \multirow{2}{*}{$(16 / 25)$} \\
\cline { 1 - 3 } $\begin{array}{c}\text { Levofloxacin } \\
\text { triple therapy }\end{array}$ & $(77.4 \%$ & $56.3 \%$ & $\mathbf{6 4 \%}$ \\
\hline
\end{tabular}

H. pylori eradication rate was $85.7 \%$ (24/28) for susceptibility-guided therapy and $53.3 \%(16 / 30)$ for empiric therapy ( $\mathrm{P}$ $=0.008)$ in the ITT populations. In the PP populations, the eradication rates was $96 \%$ (24/25) for susceptibility-guided therapy and $64 \%(16 / 25)$ with empiric therapy (P $=0.005)($ Table 4).

Table (4): Eradication rate of each group in ITT and PP analysis

\begin{tabular}{|c|c|c|c|}
\hline Analysis & $\begin{array}{c}\text { Susceptibility-guided } \\
\text { therapy }\end{array}$ & Empiric therapy & P value \\
\hline ITT $(\%, \mathbf{n} / \mathbf{N})$ & $85.7 \%(24 / 28)$ & $53.3 \%(16 / 30)$ & $<0.008$ \\
\hline PP $(\%, \mathbf{n} / \mathbf{N})$ & $96 \%(24 / 25)$ & $64 \%(16 / 25)$ & $<0.005$ \\
\hline
\end{tabular}

\section{Adverse events}

Adverse events were similar with susceptibility- guided and empiric therapies. Adverse events occurred in $21.4 \%(6 / 28)$ in susceptibility- guided therapy versus $16.6 \%(5 / 30)$ in empiric therapy $(\mathrm{P}=0.527)$. No serious adverse effects occurred and all adverse events disappeared after therapy ceased. Two subjects (1 with susceptibility- guided therapy vs 1 with empiric therapy) discontinued treatment. The most common reasons were nausea and abdominal pain (Table 5).

Table (5): Adverse events in the treatment groups

\begin{tabular}{|c|c|c|c|c|c|}
\hline Adverse events Groups & \multicolumn{2}{|c|}{$\begin{array}{l}\text { Susceptibility- } \\
\text { guided } \\
\text { therapy }(\mathrm{N}=\mathbf{2 8})\end{array}$} & \multicolumn{2}{|c|}{$\begin{array}{l}\text { Empiric therapy } \\
\qquad(\mathbf{N}=\mathbf{3 0})\end{array}$} & P-value \\
\hline No Adverse events & 22 & $78.6 \%$ & 25 & $83.3 \%$ & \multirow{7}{*}{0.527} \\
\hline Nausea & 2 & $7.1 \%$ & 2 & $6.7 \%$ & \\
\hline Abdominal pain & 1 & $3.6 \%$ & 1 & $3.3 \%$ & \\
\hline Nausea \& Abdominal pain & 1 & $3.6 \%$ & 0 & $0 \%$ & \\
\hline Diarrhea & 1 & $3.6 \%$ & 0 & $0 \%$ & \\
\hline Metallic taste & 1 & $3.6 \%$ & 0 & $0 \%$ & \\
\hline Headache & 0 & $0 \%$ & 2 & $6.7 \%$ & \\
\hline
\end{tabular}

$\mathrm{H}$ pylori eradication rates have been declining while the prevalence of antibiotic resistance has been increasing (Thung et al., 2016). Consensus 
statements recommend alternative treatment regimens to the standard triple therapy as first- line therapies; these include sequential, concomitant, or bismuth containing quadruple therapy in regions with high levels of antimicrobial resistance (Fallone et al., 2016 and Malfertheiner et al., 2017). These alternative treatments usually consist of three partly unnecessary antibiotics, thus promoting the overuse of antibiotics which fuels antimicrobial resistance (Dang et al., 2017).

Although the new treatments achieve better cure rates than triple therapy, first- line treatment of $\mathrm{H}$ pylori still fails in approximately $10 \%-20 \%$ of patients (Malfertheiner et al., 2017). Furthermore, antimicrobial resistance could be increased after first-line and second-line antibiotic use. The incidence of secondary resistance is dependent on the type of primary eradication therapy used (Park et al., 2014). However, antimicrobial susceptibility-guided therapy could avoid such problems if applied as a first- line treatment.

Our study aimed to compare the efficacy of both the antimicrobial susceptibility "culture" guided and the standard empirical triple therapy as a firstline for $\mathrm{H}$ pylori eradication. Our study demonstrated that culture guided therapy was more effective than the empirical triple therapy; the per-protocol (PP) eradication rates were $96 \%$ in the antimicrobial culture-guided group and $64 \%$ in the empirical triple therapy group.

These results are consistent with several studies as reported by Rew et al (2013) who demonstrated that susceptibility-guided therapy was more effective than standard triple therapy (94\% vs. $68 \%$ in intention-to-treat analysis) in populations with high rates of antibiotic resistance.

Also, other several studies have reported that tailored treatment based on antimicrobial susceptibility testing can increase the efficacy of first-line and rescue therapies (Smith et al., 2014 and López et al., 2015). The eradication rates of susceptibility guided treatments are generally higher than those of empirical treatment regimens (Cammarota et al., 2014 and Puig et al., 2016). Recent studies have also shown that susceptibility based treatment improves the efficacy of the therapy used after first or second-line treatment failure as well as the efficacy of first line therapy (Park et al., 2014 and Cosme et al., 2016).

The first meta-analysis comparing culture-guided triple therapy versus standard triple therapy for the treatment of H. pylori infection demonstrated that culture guided triple therapy was more effective than standard triple therapy (93\% vs $76 \%$ in PP analysis) for first-line treatment of $\mathrm{H}$. pylori infection (Wenzhen et al., 2010).

Another recent meta-analysis demonstrated that the efficacy of the first line tailored therapy was higher than that of first-line empirical therapies (Chen et al., 2016).

Our study demonstrated high rates of resistance to metronidazole, clarithromycin, amoxicillin (78.6\%, $32.1 \%$, and $32.1 \%$ respectively), While levofloxacin resistance was as low as $7.1 \%$. Resistance rates to tetracycline, nitrofurantoin and rifampicin were $17.9 \%$, $17.9 \%$, and $21.4 \%$ respectively. 
These resistance patterns greatly differ from those previously published by other Egyptian studies. Also, there is a wide discrepancy between the studies, some reported high rates of resistance to different antibiotics and others reported low rates of resistance, for example, Hasanein et al. (2011) and Fathi et al. (2013) reported $91 \%$ and $100 \%$ resistance rates to metronidazole respectively while other studies reported as low as $12.8 \%$ resistance rates (Zaki et al., 2016). Some reported low resistance rates as $6.7 \%$ to clarithromycin (Diab et al., 2018) in contrast to Fathi et al. (2013) and Zaki et al. (2016) who reported very high resistance rates.

The high rates of clarithromycin resistance demonstrated by our study (32.1\%) besides the resistance rates reported by the previous Egyptian studies could put Egypt in the zone of high clarithromycin resistance by an average of $35 \%$. Hence, the standard first-line triple therapy might be replaced by other more complex regimens.

The present study had some limitations: it was very difficult to cultivate $H$. pylori and to perform the subsequent antibiotic sensitivity testing. $\mathrm{H}$. pylori culturing is a complex process that needs a standard of quality (in terms of both materials used for culture and skill of the microbiologist to grow $\mathrm{H}$. pylori) (Chey et al., 2017). The medical cost was much higher for the tailored therapy than for empirical therapies. The rates of culture failure were high; this might reduce the applicability and effectiveness of SGT in clinical practice (López et al., 2015).
A culture-based eradication strategy demonstrated superior eradication efficacy than empirical therapy as a first-line therapy even in a region with high levels of antimicrobial resistance. It could be more effective for patients who need more reliable treatment results. In spite of the increased cost and methodological difficulty, culture- based eradication treatment could be an outstanding method from the standpoints of efficacy and safety. It is the ideal method for improving the cure rates of treatment and for preventing the emergence of antibiotic resistance, including multidrug resistance.

\section{REFERENCES}

1. Camargo, M. C., García, A., Riquelme, A., Otero, W., Camargo, C. A., Hernandez-García, T. and Rabkin, C. S. (2014): The problem of Helicobacter pylori resistance to antibiotics: a systematic review in Latin America. The American Journal of Gastroenterology, 109(4): 485-506.

2. Cammarota, G., Ianiro, G., Bibbò, S., Di Rienzo, T. A., Masucci, L., Sanguinetti, $M$ and Gasbarrini, $A$. (2014): Culture-guided treatment approach for Helicobacter pylori infection: review of the literature. World Journal of Gastroenterology, 20(18): 5205-5234.

3. Chen, H., Dang, Y., Zhou, X., Liu, B., Liu, S. and Zhang, G. (2016): Tailored therapy versus empiric chosen treatment for Helicobacter pylori eradication: a meta-analysis. Medicine, 95(7): 112-138

4. Chey WD, Leontiadis GI and Howden CW (2017): ACG clinical guide- line: treatment of Helicobacter pylori infection. Am J Gastroenterol: 112:212- 239.

\section{CONCLUSION}


5. Cosme, A., Lizasoan, J., Montes, M., Tamayo, E., Alonso, H., Mendarte, U. and Bujanda, L. (2016): Antimicrobial susceptibility-guided therapy versus empirical concomitant therapy for eradication of Helicobacter pylori in a region with high rate of clarithromycin resistance. Helicobacter, 21(1): 29-34.

6. Dang, B. N and Graham, D. Y. (2017): Helicobacter pylori infection and antibiotic resistance: a WHO high priority? Nature Reviews Gastroenterology \& Hepatology, 14(7): 383-391.

7. Diab, M., El-Shenawy, A., ElGhannam, M., Salem, D., Abdelnasser, M., Shaheen and Saber, M. (2018): Detection of antimicrobial resistance genes of helicobacter pylori strains to clarithromycin, metronidazole, amoxicillin and tetracycline among Egyptian patients. Egyptian Journal of Medical Human Genetics, 19(4): 417423.

8. Fallone CA, Chiba $\mathbf{N}$ and van Zanten SV (2016): The Toronto consen- sus for the treatment of Helicobacter pylori Infection in adults. Gastroenterology, 151:51- 69.

9. Fathi, M. S., El-Folly, R. F., Hassan, R. A. and El-Arab, M. E. (2013): Genotypic and phenotypic patterns of antimicrobial susceptibility of Helicobacter pylori strains among Egyptian patients. Egyptian Journal of Medical Human Genetics, 14(3): 235246.

10. Hasanein, W. A. G., Elsayed, H. M., Hafez, R. A. E. and Basha, O. M. (2011): Antibiotic Resistance and Failure of Eradication of Helicobacter Pylori in Egypt. Egyptian Journal of Medical Microbiology, 20(2): 30-35.

11. Lee, J. W., Kim, N., Nam, R. H., Lee, S. M., Kwon, Y. H., Sohn, S. D. and
Jung, H. C. (2019): Favorable outcomes of culture- based Helicobacter pylori eradication therapy in a region with high antimicrobial resistance. Helicobacter, 24(2): 125-141.

12. López-Góngora, S., Puig, I., Calvet, X., Villoria, A., Baylina, M., Munoz, N., and Gisbert, J. P. (2015): Systematic review and meta-analysis: susceptibilityguided versus empirical antibiotic treatment for Helicobacter pylori infection. Journal of Antimicrobial Chemotherapy, 70(9): 2447-2455.

13. Malfertheiner $P$, Megraud $F$ and O'Morain CA (2017): Management of Helicobacter pylori infection- the Maastricht V/Florence consensus report. Gut, 66: 6- 30.

14. Park, C. S., Lee, S. M., Park, C. H., Koh, H. R and Jun, C. H (2014): Pretreatment Antimicrobial Susceptibility-Guided Vs. Clarithromycin-Based Triple Therapy for Helicobacter pylori Eradication in a Region With High Rates of Multiple Drug Resistance. American Journal of Gastroenterology, 109(10): 1595-1602.

15. Puig, I., López-Góngora, S., Calvet, X., Villoria, A., Baylina, M., SanchezDelgado, J. and Gisbert, J. P. (2016): Systematic review: third-line susceptibility-guided treatment for Helicobacter pylori infection. Therapeutic advances in gastroenterology, 9(4): 437-448.

16. Rews J-S, Park SY and Ki HS (2013): An antimicrobial susceptibility guided versus standard triple therapy for Helicobacter pylori eradication in patient with gastric epithelial neoplasm. Gastroenterology; 144 (Suppl 1): S331.

17. Smith, S. M., O'Morain, C., and McNamara, D. (2014): Antimicrobial susceptibility testing for Helicobacter 
pylori in times of increasing antibiotic resistance. World Journal of Gastroenterology, 20(29): 9912-9943.

18. Thung I, Aramin $H$ and Vavinskaya $V$ (2016): Review article: the global emergence of Helicobacter pylori antibiotic resistance. Aliment Pharmacol Ther, 43:514- 533.

19. Wenzhen, Y., Yumin, L., Quanlin, G., Kehu, Y., Lei, J., Donghai, W. and Lijuan, Y. (2010): Is antimicrobial susceptibility testing necessary before first-line treatment for Helicobacter pylori Infection?-Meta-analysis of randomized controlled trials. Internal Medicine, 49(12): 1103-1109.
20. Zaki, M. E., Sherif, D. M., Ali, M. A., Shehta, A., Megahed, A., Alsayed, M. A. L. and Elzeny, S. M. (2016): Molecular study of primary clarithromycin resistant Helicobacter pylori strains from Egyptian centre. Int. J. Curr. Microbiol. App. Sci, 5(1): 165173.

21. Zaki, M., Othman, W., Ali, M. A. and Shehta, A. (2016): Fluoroquinoloneresistant Helicobacter pylori strains isolated from one Egyptian University Hospital: molecular aspects. J Microbiol Antimicrobial Agents, 2: 26-31. 


\section{تقييم العلاج التجريبي مقابل العلاج القائم على الحساسية في

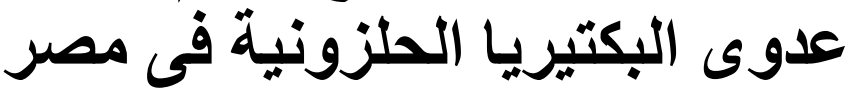

عمرو هانى الجزار، محمد على البرعي، سوسن هانم محمد الطيبث، عادل عبد الفتاح الركيب

قسمى الأمراض الباطنة والباثولوجيا الاكلينيكية*، كلية الطب، جامعة الأزهر

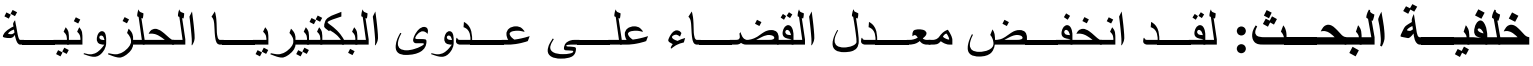
فى مصر ويرجع ذلك الى مقاومة هذة البكتيريا الى المضادات الحيوية.

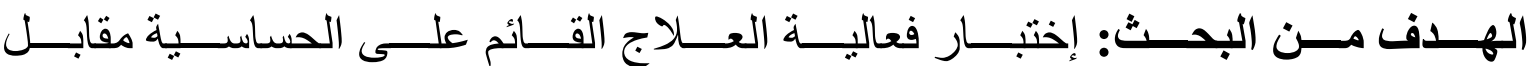
العلاج الثناثي التجريبي كخط اول لعلاج عدوى البكتيريا الحلزونية.

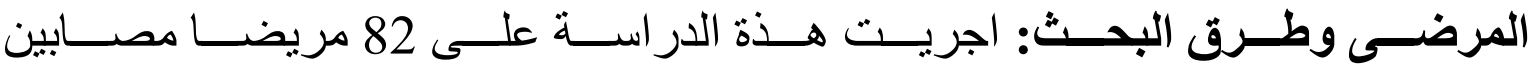

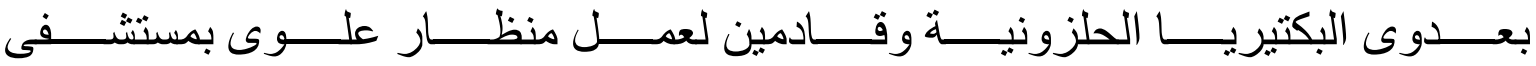

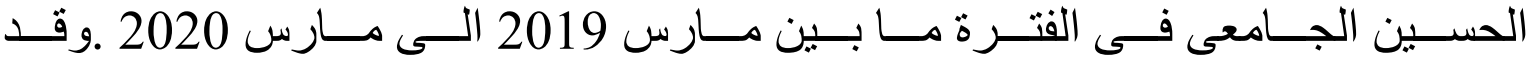

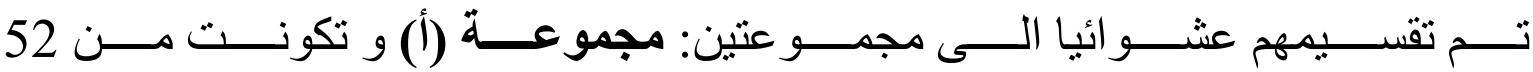

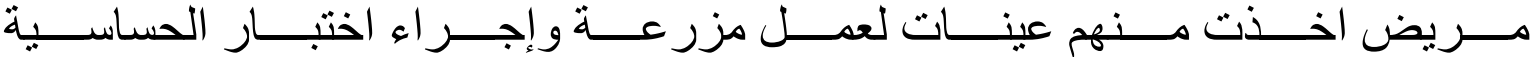

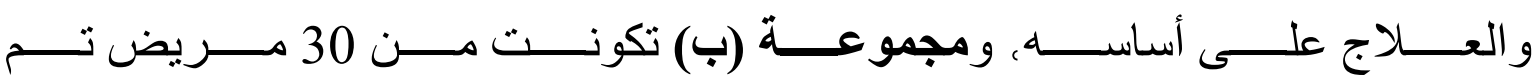

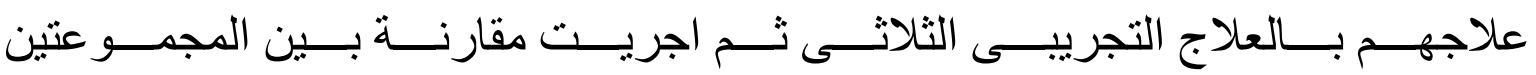
من حيث فاعلية القضاء على العدوى والأعر اض الجانبية.

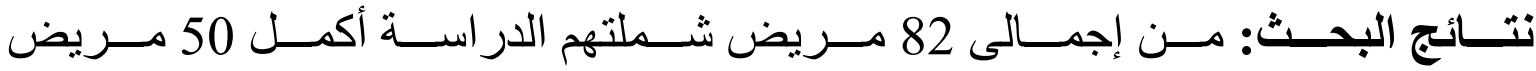

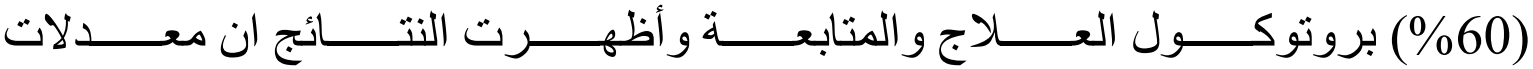

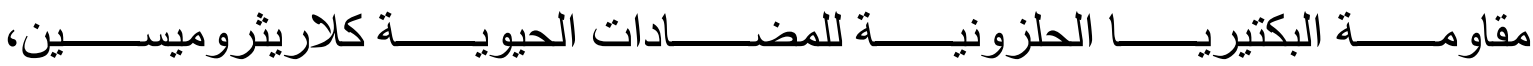

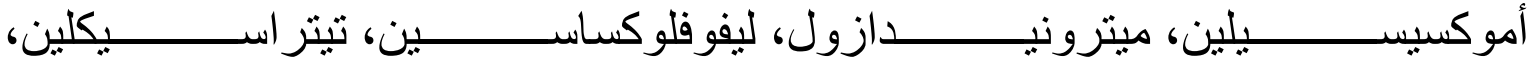

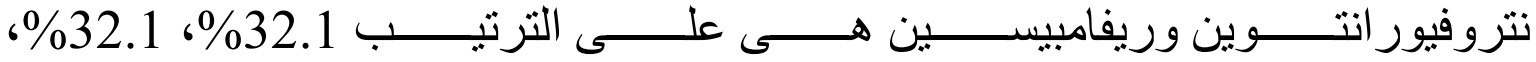

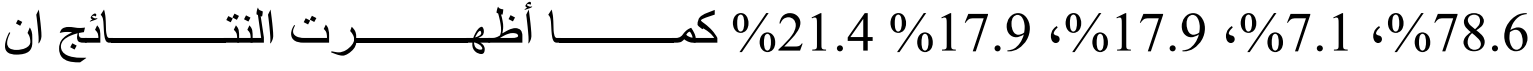




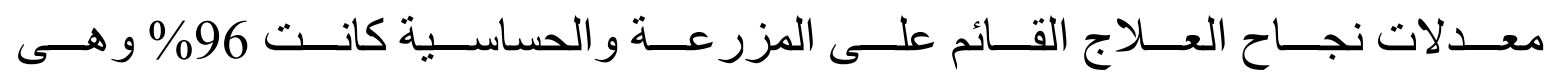

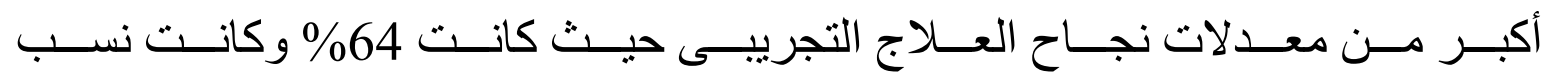

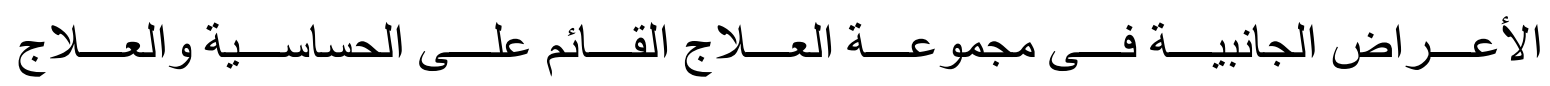
التجريبى الثناثى هى على الترتيب (21.4\% و 16.6\%).

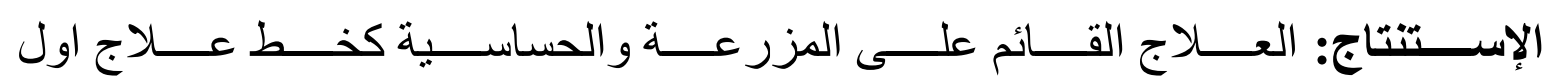

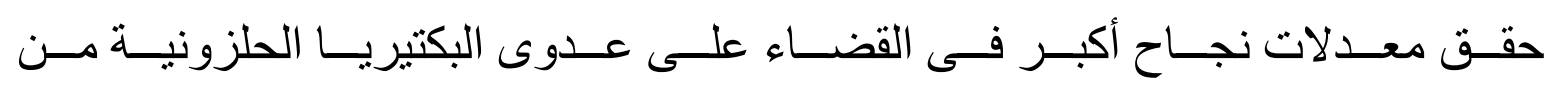
العلاج التجريبى الثلاثى. 